\title{
Über den Einfluss der Strömungsgeschwindigkeit auf die Selbstreinigung in Fliessgewässern
}

\author{
Von K. WuhrmanN, E. Eichenberger, H.A.Leidner und D. WüEst
}

Eidg. Tech. Hochschule, Institut für Gewässerschutz und Wassertechnologie (IGW), und Eidg. Anstalt für Wasserversorgung, Abwasserreinigung und Gewässerschutz (EAWAG)

Manuskript eingegangen am 2. Juli 1975

\section{ABSTRACT}

Flow Velocity as a Rate Determining Factor for Selfpurification in Rivers

Experiments in model rivers on selfpurification in function of the concentrations of pollutants and of flow velocity have indicated that the ratio of the rates of substrate transports from the flowing wave on to cell surfaces and of active transports in substrate uptake is a determining factor for selfpurification rates. This ratio is decreasing rapidly with decreasing flow velocities of the water. The results were discussed on the basis of the kinetics of active transports.

\section{Problemstellung und Versuchsfragen}

Die vorliegende Untersuchung beschäftigt sich mit Selbstreinigungsprozessen in Fliessgewässern, die in der Elimination gelöster organischer Verbindungen durch mikrobielle Stoffwechselprozesse bestehen. Wie früher gezeigt wurde [1], sind in diesem Rahmen erwartungsgemäss fast nur die heterotrophen Phytozönosen als Reaktionspartner zu betrachten. Phototrophe Mikrophyten in natürlichen Gewässern nehmen nur unwesentliche Mengen organischer Substrate auf.

Die Resorptionsleistung einer aggregierten Biomasse, wie sie beispielsweise im Bodenbewuchs eines Baches vorliegt, ist von der Geschwindigkeit des Transports der Substrate aus der fliessenden Welle an die Rezeptorstellen an der Oberfläche der einzelnen Zellen abhängig. Da man es in der Natur häufig mit Substratkonzentrationen (Verunreinigungen) zu tun hat, welche weit unter den Konzentrationen für eine Sättigung der Systeme für aktive Transporte bei der Substratresorption liegen, wird die Transportgeschwindigkeit im Umgebungsmilieu der Zellverbände erst recht ein begrenzender Faktor für die Selbstreinigung. Für die Substratversorgung der Zellen einer festsitzenden Kolonie von z.B. Sphaerotilus natans sind damit zwei Faktoren zu berücksichtigen: a) die Umspülung der Bakterienkolonien mit der Substratlösung (turbulente Diffusion) und b) die Diffusion von Substraten zu den einzelnen Zellen durch das Interstitialsystem innerhalb der Kolonie (vorwiegend molekulare Diffusion). Das massgebende Konzentrationsgefälle für diese Transporte ist gegeben 
durch die Konzentrationen in der fliessenden Welle einerseits und an den Rezeptorstellen der Zellen anderseits [3]. Der zweite Prozess ist natürlich geschwindigkeitsbegrenzend, doch kommt der Wasserströmung trotzdem eine massgebende Bedeutung $z u$, da sie für die Expansion und die Exposition aggregierter, flockiger oder fellartiger Organismenaufwüchse (Sphaerotilus, Leptomitus, Fusarium) oder strähniger Phytozönosen (Fadenalgen oder Diatomeen mit ihren heterotrophen Epiphyten) in der fliessenden Welle sorgt.

Eine weitere Wechselwirkung zwischen Strömungsgeschwindigkeit und Selbstreinigung entsteht mit der strömungsbedingten Begrenzung der maximal möglichen stehenden Biomasse in einem Flussbett, die ja von den drei Prozessen Zuwachsgeschwindigkeit, Predation und Abtrieb reguliert wird. Die minimale stehende Biomasse und damit das Minimum der Selbstreinigung wird bei beginnender Instabilität der Flußsohle erreicht, da dann eine Organismenakkumulation nicht mehr möglich ist.

In der vorliegenden Arbeit haben wir uns das Problem gestellt, einen Einblick in die kausalen Zusammenhänge zwischen der direkt beobachtbaren Selbstreinigung in einer Flußstrecke und der Beeinflussung der Kinetik des massgebenden Stoffwechselprozesses, d.h. der Substratresorption der beteiligten Organismen durch den Faktor Strömungsgeschwindigkeit, zu gewinnen.

\section{Arbeitshypothesen}

Da in natürlichen Fliessgewässern immer gemischte Phytozönosen vorkommen, beobachtet man bei der Messung irgendeiner physiologischen Reaktion, wie z.B. der Selbstreinigung (im Sinne der Elimination eines Substrates aus dem Milieu), einen summarischen Wert aller beteiligten Organismen. Aus solchen Messungen berechnete «kinetische Parameter» sind daher rein empirische, nur für die betreffende Biozönose geltende, globale Werte. Thre Grössen sind sowohl von physiologischen als auch von extrazellulären Faktoren beeinflusst (z.B. Diffusion in interzellulären Räumen). Wenn wir die Denkweise der biochemischen Reaktionskinetik dennoch auf solche natürlichen Systeme übertragen, so geschieht dies aus der Erfahrung, dass sich Reaktionen makroskopischer Organismensysteme damit recht gut beschreiben lassen. Einzelne externe Faktoren üben auf ganze Organismengemeinschaften die gleichen Effekte aus wie auf Einzelindividuen, so dass insbesondere die dominanten geschwindigkeitsbegrenzenden Faktoren für Reaktionen ganzer Gemeinschaften auch bei globaler Betrachtung sichtbar zu machen sind.

Wir verwenden in den weiteren Ausführungen folgende Definitionen und Bezeichnungen: 1. Biomasse ist die Gesamtheit der auf einer Gewässersohle pro Flächeneinheit vorhandenen Organismen. In unseren Experimenten handelt es sich fast ausschliesslich um Phytozönosen. 2. Substrate sind in unseren Experimenten Mischungen, die aus bekannten Einzelkomponenten zusammengesetzt sind und individuell analysiert werden. Aus Dimensionsgründen werden ihre Konzentrationen häufig als org. $\mathrm{C}$ angegeben (wenn sie in Beziehung mit Biomasse gesetzt werden, die auch als org. $\mathrm{C}$ angegeben wird). 3. Nachstehende Symbole gehen in unsere algebraischen Formeln ein: 
$\mu, \mu_{m}$ Effektive bzw. maximale Wachstumsgeschwindigkeit (Zunahme der Biomasse) [Zeit $\left.{ }^{-1}\right]$

$V, V_{m}$ Effektive bzw. maximale Resorptionsrate eines Substrates durch die Biomasse

[Zeit-1]

$s_{0}, s_{z}$ Substratkonzentration zu Anfang und Ende einer Selbstreinigungsstrecke $\left[\mathrm{mg} \mathrm{l}^{-1}\right]$, [ $\left.\mathrm{mol} \mathrm{l}^{-1}\right],\left[\mathrm{mg}\right.$ org. $\left.\mathrm{C}^{-1}\right]$

$x_{0}, x_{z}$ Biomasse im Gewässer am Anfang bzw. Ende einer Selbstreinigungsstrecke [mg Glühverlust $\left.\mathrm{m}^{-1}\right],\left[\mathrm{mg} \mathrm{GV} \mathrm{m}^{-2}\right]$, [mg org. $\left.\mathrm{C} \mathrm{m}^{-1}\right]$, [mg org. $\left.\mathrm{C} \mathrm{m}^{-2}\right]$, $\left[\mathrm{mg}\right.$ org. $\mathrm{C}^{-1}$ ]

$K_{m} \quad$ Halbwertskonzentration für maximale Resorptionsrate $V_{m}$ $\left[\mathrm{mg} \mathrm{l}^{-1}\right],\left[\mathrm{mol} \mathrm{l}^{-1}\right]$

$z \quad$ Fliessdistanz zwischen Anfang und Ende einer Selbstreinigungsstrecke [m]

$v \quad$ Fliessgeschwindigkeit

$\left[\mathrm{cm} \mathrm{s}^{-1}\right]$

$Q \quad$ Abflussmenge

$\left[1 \mathrm{~s}^{-1}\right]$

$S_{m} \quad$ Grösse der Selbstreinigung,

$S_{m}=Q\left(s_{0}-s_{z}\right)$

$S_{r} \quad$ Selbstreinigungsgeschwindigkeit,

$S_{r}=\left(s_{0}-s_{z}\right) t^{-1}$

$S_{e} \quad$ Spezifische Selbstreinigungsleistung einer Biomasse, $S_{e}=S_{m} / G \quad\left[\mathrm{~mol} \mathrm{~g}^{-1} \mathrm{~s}^{-1}\right]$, d.h. [Zeit-1]

$G \quad$ Gesamte Biomasse in einer Selbstreinigungsstrecke (sessil und suspendiert)

$Y \quad$ Ausnützungskoeffizient des Substrats für den Baustoffwechsel

Für die Experimente waren folgende Ziele gesetzt: a) Ermittlung von globalen Resorptionsgeschwindigkeits-Konstanten von charakteristischen Flussphytozönosen für organische Modellsubstanzen bei definierten Milieubedingungen und b) Messung des Einflusses der Strömungsgeschwindigkeit auf die Resorptionsleistung von gegebenen Phytozönosen.

\section{Methoden}

Die Freilandexperimente wurden in den sog. «kleinen» bzw. «grossen» künstlichen Gerinnen in unserer Versuchsanlage Tüffenwies vorgenommen (technische Beschreibungen dieser Modellgewässer vgl. z.B. [4]). Bei allen in dieser Arbeit beschriebenen Experimenten waren die Kanalsohlen mit Kalkgeröllen (Durchmesser 40-60 mm) in einer Schicht belegt. Die Speisung der Rinnen erfolgte mit Grundwasser aus eigenem Filterbrunnen. Es handelt sich um ein mittelhartes (Ca-Härte um 12 fr. $\mathrm{H}^{\circ}$ ), sehr reines Wasser (org. C 0,5-0,7 mg C/1). Die für die Messungen der Selbstreinigung gewünschte Besiedlung der Rinnen wurde mittels künstlicher «Verunreinigung» des Grundwassers (Dosierungen von anorganischen Salzen oder billigen organischen Substanzgemischen) induziert. Alle Dosierungen von Zusätzen zum Grundwasser in den Rinnen erfolgten mit Präzisionsmikropumpen aus Vorratsbehältern, welche konzentrierte Stammlösungen enthielten. Die chemische Kontrolle der Selbstreinigungsexperimente erfolgte mittels quantitativer Konzentrationsbestimmung jeder einzelnen Komponente in der "Verunreinigungslösung». 
Die diesbezüglichen Methoden für Zucker haben wir früher beschrieben [5], die Glutaminsäurebestimmungen erfolgten mit der konventionellen Ninhydrin-Methode. Die Biozönosen in den Rinnen wurden bei jeder Versuchsserie zunächst nach Artenzusammensetzung und Dominanzen beschrieben und sodann quantitativ gemessen. Hierzu wurde in den Selbstreinigungsstrecken die Biomasse auf $1 \mathrm{~m}$ Länge an mehreren Stellen vollständig aufgesammelt und analysiert (Frischgewicht, Trockengewicht, Glühverlust, org. C, evtl. N und P, Chlorophyll). Weitere methodische Hinweise finden sich bei der Beschreibung der einzelnen Experimente.

\section{Kinetische Daten für die Resorption (Selbstreinigung) von organischen Verbindungen durch Fliessgewässer-Biozönosen}

\subsection{Grundlagen}

Abgesehen von theoretisch nicht auszuschliessenden extrazellulären Umsetzungen von biodegradierbaren, organischen Substanzen in einem Fliessgewässer ist ihre Elimination ausschliesslich der Resorption durch die Zellen der einzelnen Mikrophytenarten der Biozönosen in einem Flusse zu verdanken. Die nach erfolgter Resorption stattfindende anabolische und katabolische Umsetzung der Substrate in den Organismen selbst ist für die Konzentrationsveränderungen im Gewässer nicht mehr von unmittelbarem Interesse. Die Geschwindigkeit der Selbstreinigung ist damit von der Kinetik der Resorptionsreaktionen bestimmt.

Die Resorption der in den vorliegenden Experimenten verwendeten Substrate erfolgt mittels aktiven Transports. Ihre Geschwindigkeit ist damit beschreibbar mit der Kinetik enzymatischer Reaktionen

$$
V=V_{m} \frac{s}{K_{m}+s}[\mathrm{~mol} \text { Substr./mol Ferment } \cdot \text { Zeit }]=\left[\text { Zeit }^{-1}\right] \text {. }
$$

Wenn man diese Kinetik auf ein Organismensystem überträgt, das hinsichtlich seiner Reaktionsweise zwar nur gesamthaft, als statistische Grösse, beurteilt werden kann, so lassen sich doch Reaktionskonstanten $V_{m}$ und $K_{m}$ für die Resorption einer Einzelsubstanz berechnen, die gewissermassen systeminhärent sind. Damit eröffnet sich die Möglichkeit, den Einfluss äusserer Faktoren (Substratkonzentrationen, Temperatur, Strömungsgeschwindigkeit usw.) auf die Resorptionsleistung eines Organismensystems mittels physiologisch relevanter Parameter zu erfassen.

Die Resorptionsgeschwindigkeit $V$ ist mit dem früher definierten Begriff der spezifischen Selbstreinigungsleistung der «Biomasse» $S_{e}$ identisch, wenn man mit einheitlichen Massen arbeitet, wie beispielsweise dem org. C für Biomasse und Substrate. Ermittelt man $S_{\boldsymbol{e}}$-Werte in ein und derselben Selbstreinigungsstrecke bei verschiedenen Konzentrationen desselben Substrates, so kann man mit der bekannten Methode von Lineweaver und Burk aus der Inversion der Beziehung (1) die Werte von $V_{m}$ und $K_{m}$ im Sinne von globalen, statistischen Eigenschaften des betreffenden Organismensystems im untersuchten Flussabschnitt ermitteln. Aus ihrer Variabilität in Funktion der Strömungsgeschwindigkeit bei sonst unveränderten ökologischen Bedingungen lässt sich dann objektiv die Bedeutung des Parameters «Strömung" für die globale Physiologie der Biozönosen abschätzen. 


\subsection{Experimentelle Ergebnisse}

Versuche entsprechend der Arbeitshypothese erfolgten im "kleinen» Rinnensystem. Die Biozönose wurde mittels Zusatz einer Lösung von Melasse und Glutaminsäure (Endkonzentration in der Rinne etwa $8 \mu M$ Saccharose bzw. $8 \mu M$ Glutaminsäure) vorgezüchtet. Es bildete sich unterhalb der Verunreinigungsquelle (Dosierungsstelle der Melasselösung) eine zweischichtige Phytozönose aus: ein krustiger Bewuchs auf der Rinnensohle aus vorwiegend Diatomeen und darüber eine dichte, flutende Masse sessiler heterotropher Mikrophyten (dominant Sphaerotilus natans mit seinen Kommensalen). Dieses obere "Stockwerk» der Biozönose wies erwartungsgemäss einen grossen Massegradienten in den ersten $200 \mathrm{~m}$ der Fliessstrecke auf und zeigte schon rein äusserlich den raschen Fortgang der Selbstreinigung im Sinne der Elimination der zugefügten organischen Verbindungen an. Die Strecke von 1,6 m bis 66,6 m ab Dosierungsstelle, welche für das Experiment ausgewählt wurde, enthielt trotz deutlichem Biomassegradienten eine relativ homogene Biozönose. Am Versuchstag wurde vorerst die Dosierung des Wachstumssubstrates eingestellt. Nach Durchspülung der Experimentierstrecke mit Grundwasser wurde das Testsubstrat in Form einer Stammlösung mit Glukose, Fructose, Saccharose und Glutaminsäure je während 1 Stunde in 3 verschiedenen Dosierungen dem Grundwasser zugesetzt. Die Selbstreinigung wurde bei jeder Dosierungsintensität an 3

Tabelle 1. Selbstreinigung im selben Biotop nach unterschiedlicher Belastung mit Zuck',rn. Table 1. Selfpurification in the same biotope following various sugar loadings.

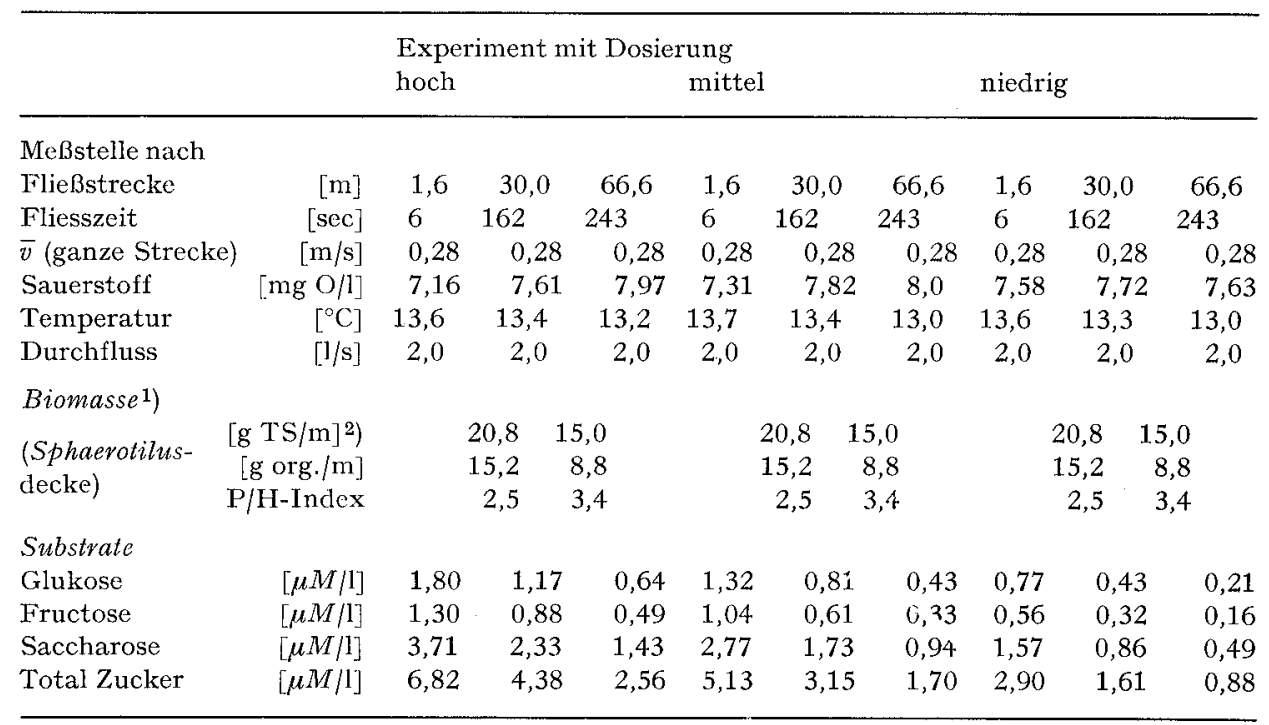

1) Kompakte flutende Sphaerotilusdecke über krustigem Sohlenbewuchs aus Phormidium autumnale, Achnanthes- und Nitzschia-Arten.

2) $1 \mathrm{~m}$ Rinnenlänge entspricht etwa $0,4 \mathrm{~m}^{2}$ benetzter, besiedelter Fläche.

1) Compact cover of Sphaerotilus streamers on top of crusty growth consisting of Phormidium autumnale, Achnanthes and Nitzschia sp.

2) $1 \mathrm{~m}$ of the model river corresponds to about $0.4 \mathrm{~m}^{2}$ of wetted and settled surface. 
Probenahmestellen $(1,6 \mathrm{~m}, 30 \mathrm{~m}, 66,6 \mathrm{~m})$ mittels 4 Probenahmen im Abstand von 15 Minuten verfolgt (total für ganzes Experiment also $3 \times 4 \times 3$ Proben, 3 Parallelanalysen pro Substanz, d.h. pro Substanz $108-1=107$ Freiheitsgrade). Nach Versuchsabschluss wurde die heterotrophe Biomasse über dem Krustenbewuchs quantitativ an je 2 Stellen zwischen den Probenahmepunkten abgeerntet und analysiert (total 4 Probenahmestrecken von je $1 \mathrm{~m}$ Länge). Tabelle 1 enthält alle wesentlichen Beobachtungen, gegliedert nach den 3 Dosierungsintensitäten des Testsubstrates (hoch: etwa $7 \mu M$, mittel: etwa $5 \mu M$ und niedrig: etwa $3 \mu M$ Gesamtzucker).

\subsection{Geschwindigkeitskonstanten für die Zuckerelimination}

Trägt man die Logarithmen der Zuckerkonzentrationen im Längsprofil der Selbstreinigungsstrecke für die 3 Versuchsreihen auf, so ergibt sich eine streng lineare Regression mit sehr geringen Streuungen (Varianz der Regression für alle Substanzen $\ll 5 \%$ ), d.h. das Konzentrationslängsprofil kann mit einer $e$-Funktion

$$
\ln \frac{s_{z}}{s_{0}}=-k \cdot z
$$

recht genau angenähert werden. Da die stehende heterotrophe Biomasse mit der vorhandenen Substratkonzentration an jeder Stelle korreliert ist, darf man auch für sie eine exponentielle Massenverteilung (allerdings mit grosser Streuung) innerhalb der Meßstrecke approximieren. Wir haben innerhalb der ganzen Beobachtungsstrecke 3 Teilstrecken von $10 \mathrm{~m}$ Länge, d. h. 0-10 m, 25-35 m und 55,6-66,6 m, für die weiteren Berechnungen ausgewählt. Aus den gefundenen Konzentrationsgradienten für

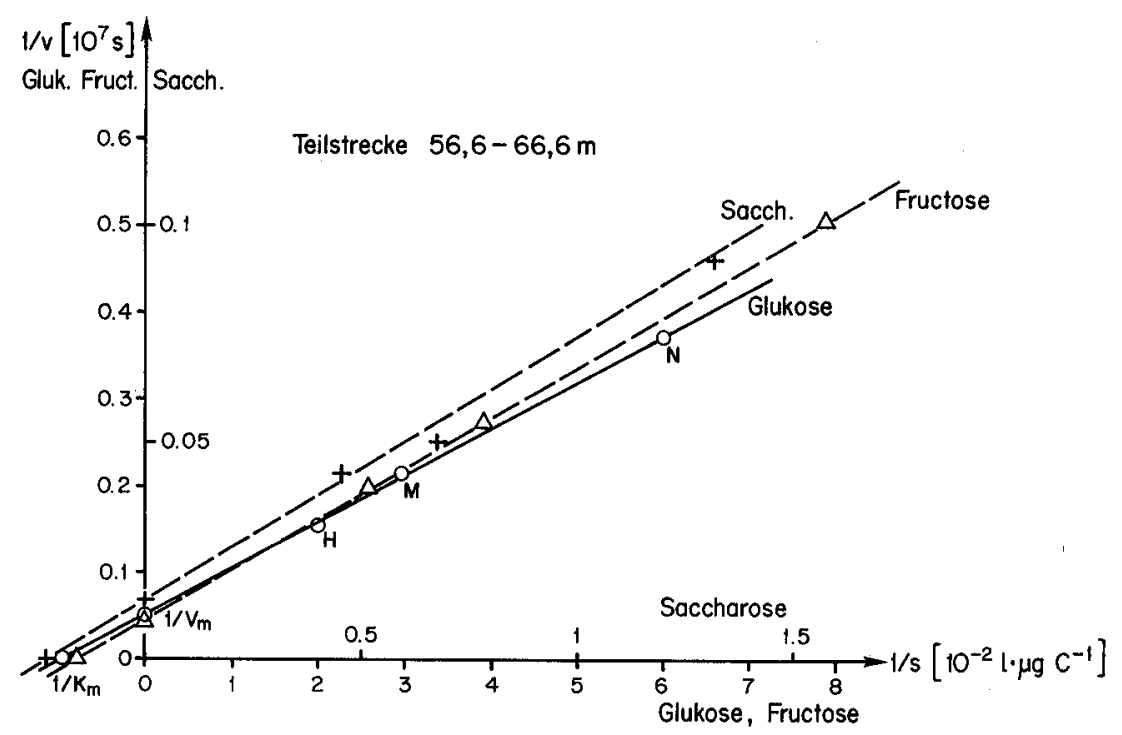

Abb. 1. Regression von $1 / V$ gegen $1 / s$ für die Teilstrecke 55,6-66,6 $\mathrm{m}$ (Mittelwert von $s_{O}$ und $s$ bzw. $x_{0}$ und $x$ als Rechnungsbasis genommen).

Fig. 1. Regression of $1 / V$ on $1 / s$ for the section 55.6-66.6 m (average values of $s_{0}$ and $s, x_{0}$ and $x$ respectively are used as a basis for calculation). 
Substrate und Biomasse konnten rechnerisch die Substrat- und Biomassekonzentrationen für den Anfang und das Ende dieser Teilstrecken ermittelt werden. Aus diesen Werten ergab sich für jede Substratdosierung die Grösse der Selbstreinigung $S_{m}$ und die spezifische Selbstreinigungsleistung $S_{e}$ oder $V\left(\right.$ Zeit $\left.^{-1}\right)$. Man erhält dann für die Teilstrecke um jede Probenahmestelle 3 Werte von $V$ und drei zugehörige Werte der Substratkonzentration (Mittelwert von $s_{0}$ und $s_{z}$ ). Diese Wertepaare gestatten die Ermittlung der Resorptionskonstanten $V_{m}$ und $K_{m}$. Abb. 1 gibt als Beispiel für die 3 Zucker eine Lineweaver-Burk-Darstellung der Verhältnisse in der untersten Teilstrecke (massgebendes $\vec{s}=$ Konzentrationsmittelwert bei 55,6 und 66,6 $\mathrm{m}$ ). Die sehr guten linearen Regressionen, welche für alle Teilstrecken und alle Zucker gefunden wurden, zeigen, dass das Prinzip dieser Auswertung durchaus brauchbar

Tabelle 2. Kinetische Konstanten für die Zuckerresorption der Rinnenbiozönosen, berechnet aus den Eliminationsmessungen. $\mathrm{T}=13,5^{\circ} \mathrm{C}$. Annahme: Resorption $=$ aktiver Transport, wobei $V=V_{m} \frac{s}{K_{m}+s}$.

Table 2. Kinetic constants for sugar resorption by the biocenosis in the model rivers calculated on the basis of elimination measurings. $\mathrm{T}=13.5^{\circ} \mathrm{C}$. Assumption : Resorption $=$ active transport whereby

$V=V_{m} \frac{s}{K_{m}+s}$.

\begin{tabular}{llrrrr}
\hline Strecke & & Glukose & Fructose & Saccharose & Tot. Zucker \\
\hline $0-10 \mathrm{~m}$ & $V_{m} 10^{-7}\left[\mathrm{~s}^{-1}\right]$ & 14 & 25 & 71 & 142 \\
& $K_{m}\left[\mu \mathrm{C} \mathrm{C1^{-1 } ]}\right.$ & 213 & 498 & 1333 & 2702 \\
$25-35 \mathrm{~m}$ & $V_{m} 10^{-7}\left[\mathrm{~s}^{-1}\right]$ & 16 & 28 & 66 & 126 \\
$56,6-66,6 \mathrm{~m}$ & $K_{m}\left[\mu \mathrm{C} \mathrm{C}^{-1}\right]$ & 162 & 340 & 750 & 1410 \\
& $V_{m}^{*} 10^{-7}\left[\mathrm{~s}^{-1}\right]$ & 19 & 22 & 74 & 120 \\
& $K_{m}^{*}\left[\mu \mathrm{g} \mathrm{C1^{-1 } ]}\right.$ & 105 & 128 & 444 & 719 \\
\hline
\end{tabular}

$V_{m}^{*}, K_{m}^{*}=$ Physiologisch günstigste, beobachtete Werte (für weitere Berechnungen verwendet in der Auswertung der Experimente über Einfluss der Fliessgeschwindigkeit).

$V_{m}^{*}, K_{m}^{*}=$ Physiologically most favorable values (used for further calculations in the evaluation of the experiments on the influence of flow velocity).

ist, obschon es sich zugegebenermassen um eine ungewöhnliche Übertragung eines enzymkinetischen Messprinzips auf ein natürliches Ökosystem handelt. Die mit dieser Methode gefundenen und in Tabelle 2 vereinigten $V_{m}$ - und $K_{m}$-Werte sind bekanntlich gegenüber kleinsten Ungenauigkeiten der Wertepaare $s$ und $V$ sehr empfindlich. Trotzdem sind die Streuungen der in unserem Experiment für jeden einzelnen Zucker (die Glutaminsäure wurde nicht analysiert) ermittelten Grössen dieser Konstanten erfreulich gering.

Erwartungsgemäss sind die $V_{m}$-Werte für alle Teilstrecken annähernd von gleicher Grösse und nur vom Substrat abhängig. Auffällig ist hingegen der starke Abfall von $K_{m}$ innerhalb der Selbstreinigungsstrecke, der mit der Abnahme der Biomassemenge pro Flächeneinheit Rinnensohle parallel verläuft (Abb. 2). Obschon zwar $K_{m}$ und $V_{m}$ substrat- und organismenspezifische Transportkonstanten dar- 
stellen, ist leicht einzusehen, dass sich ihre Globalwerte in unserem Ökosystem unterschiedlich verhalten müssen: $V_{m}$ ist ein hypothetischer Maximalwert der Resorptionsgeschwindigkeit einer Zelle für eine bestimmte Substanz, deren Grösse bei gleichartigen Milieubedingungen unveränderlich ist. $K_{m}$ ist hingegen die Substratkonzentration, bei der $V=V_{m} / 2$ ist, d.h. eine Grösse, welche von den experimentellen Bedingungen bezüglich der extrazellulären Transporte abhängt.

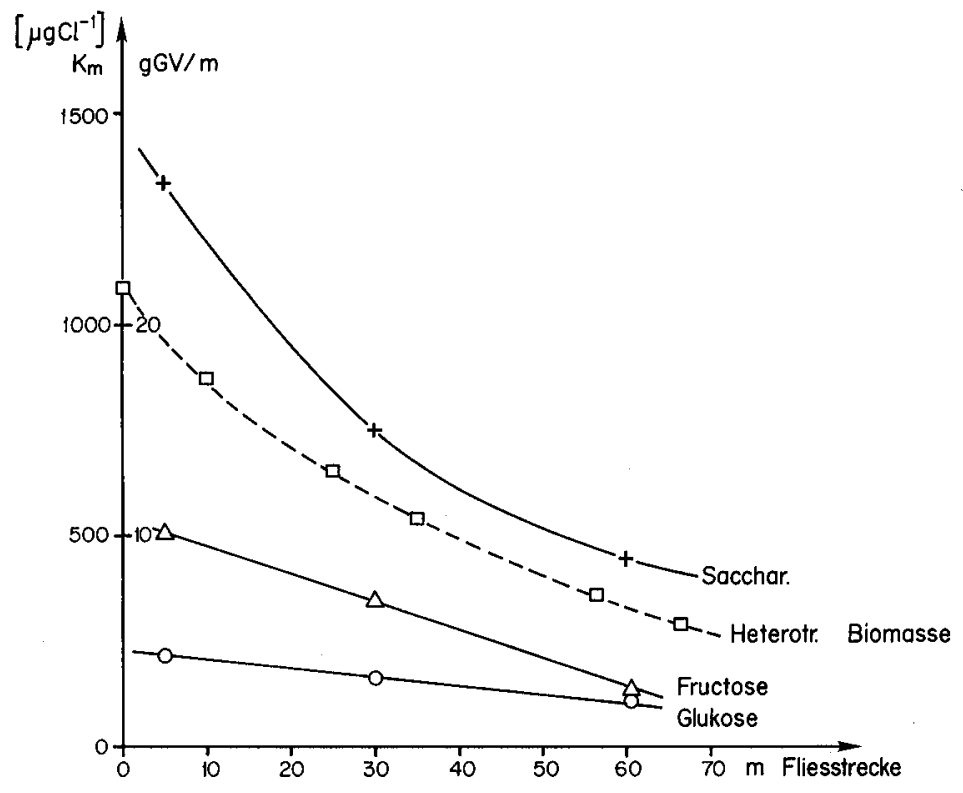

\begin{abstract}
Abb. 2. $K_{m}$-Werte für die Zuckerresorption in Funktion der Fliessdistanz von der Dosierungsstelle. Die ebenfalls eingezeichnete Kurve der Biomasse wurde aus den an 4 Stellen gemessenen Werten rechnerisch ermittelt.

Fig. 2. $K_{m}$-values for the resorption of sugars in function of the flow distance from the point of dosage.
\end{abstract}

Wir betonen nochmals, dass die gefundenen $V_{m}$ und $K_{m}$-Werte vom biochemischen Standpunkt aus gesehen fiktive, summarische Werte für die Gesamtheit der Heterotrophen in den Rinnenbiomassen darstellen, aber keinesfalls als enzymkinetische Reaktionskonstanten im engeren Sinne missbraucht werden dürfen! Sie gestatten aber trotzdem die Ermittlung von Relativwerten für Einflüsse von Ökofaktoren auf die Resorptions-, d.h. Selbstreinigungsgeschwindigkeiten.

\title{
5. Einfluss der Strömungsgeschwindigkeit auf Selbstreinigungsvorgänge
}

Die beschriebenen kinetischen Experimente geben bereits mit der Beobachtung der Abhängigkeit von $K_{m}$ von der Menge der Biomasse im Gerinne einen Hinweis auf die Bedeutung der Wasserströmung für Substrattransporte innerhalb der Organismengemeinschaft auf einer Flußsohle. Mit ihrer Hilfe konnte nun eine systematische Prüfung dieses Faktors für die Selbstreinigung erfolgen.

\subsection{Experimentelles Vorgehen}

In den "grossen" Rinnen wurden mit üblicher Technik drei Biozönosen gewünschten Aufbaus mittels Zusätzen zum Grundwasser induziert. Experimentelle Ein- 
Tabelle 3. Phytozönosen zur Zeit der Selbstreinigungsversuche mit verschiedenen Fliessgeschwindigkeiten.

Table 3. Phytocoenoses at the time of selfpurification experiments with varying flow velocities.

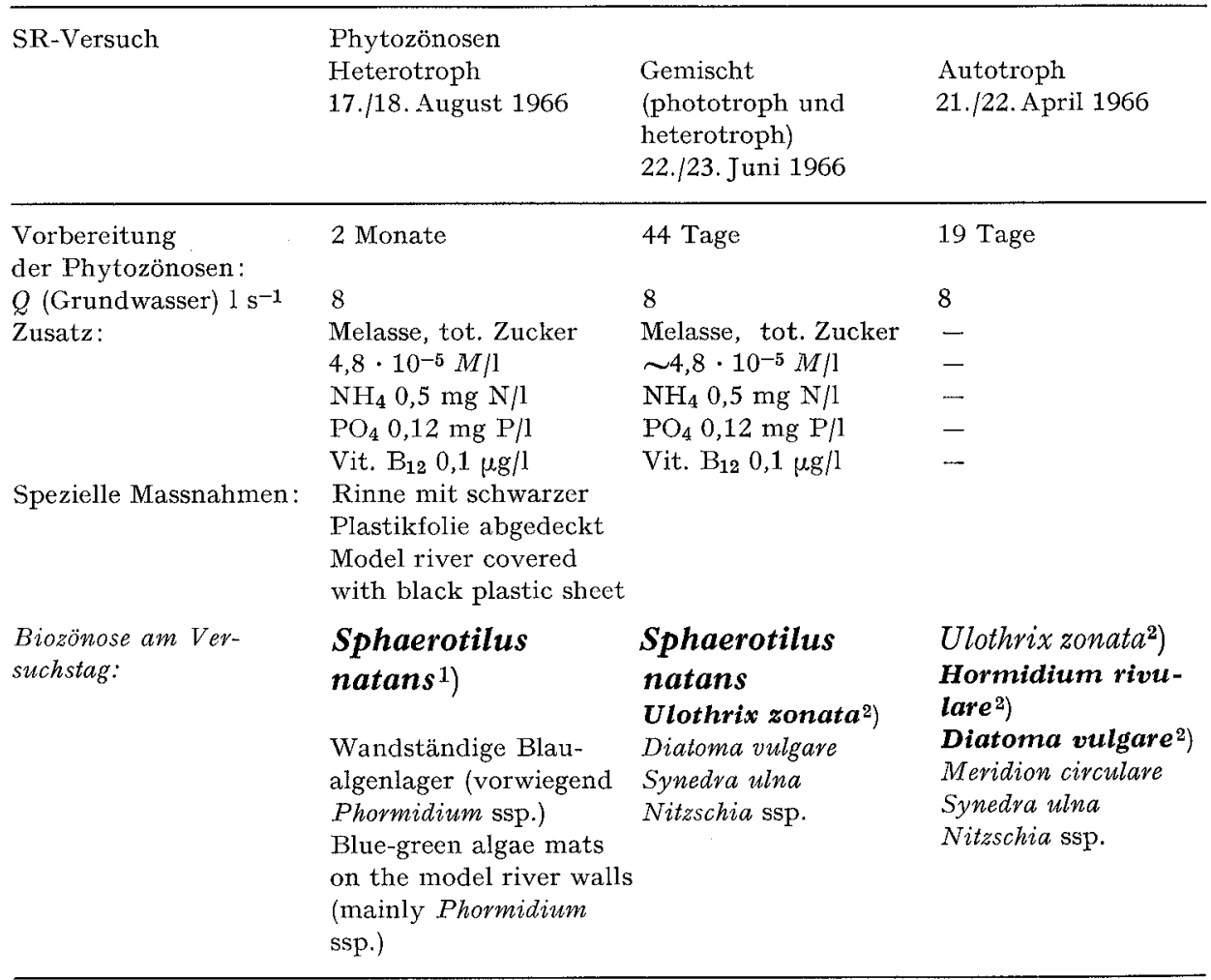

Dichteschätzung: $\left.{ }^{1}\right)$ Mehr als $1 / 2$ der Bewuchsmasse; ${ }^{2}$ ) mehr als $1 / 4$ der Bewuchsmasse.

Estimate of growth density: 1 ) More than $1 / 2$ of the biomass; ${ }^{2}$ ) more than $1 / 4$ of the biomass.

zelheiten und biologische Charakteristika der Phytozönosen zur Zeit der nachstehend beschriebenen Selbstreinigungsversuche sind in Tabelle 3 zusammengefasst. Für die Bestimmung des Einflusses der Wasserströmung wurde wie folgt vorgegangen: Es wurden 3 Fliessgeschwindigkeiten geprüft, welche durch die Abstufung der Zuflussmenge auf 8, 4 und $11 \mathrm{~s}^{-1}$ erreicht wurden. Da die Vorzucht der Biomassen bei einem Rinnenfüllungsgrad entsprechend der Dosierung von $8 \mathrm{l} \mathrm{s}^{-1}$ erfolgt war und es entscheidend darauf ankam, bei allen Fliessgeschwindigkeiten die gesamte Biomasse in Kontakt mit der fliessenden Welle zu halten, wurden bei den Abflussmengen von 4 bzw. $11 \mathrm{~s}^{-1}$ mittels künstlichen Rückstaus die Wasserspiegel in den Rinnen auf einheitliche Höhe reguliert (Einsetzen von Staublechen im Längsprofil der Teststrecke). Die effektive Fliessgeschwindigkeit während der Experimente wurde mittels Messung der Verschiebungsgeschwindigkeit des Konzentrationsmaximums eines KCl-Stosses ermittelt (konduktometrische Bestimmung). Die Stammlösung mit 
Tabelle 4. Beobachtungen in Selbstreinigungsversuchen mit verschiedenen Fliessgeschwindigkeiten.

Table 4. Observations made in selfpurification experiments with varying flow velocities.

\begin{tabular}{|c|c|c|c|c|c|c|c|c|c|c|c|}
\hline & & & \multicolumn{3}{|c|}{$\begin{array}{l}\text { Heterotrophe } \\
\text { Biozönose } \\
\mathrm{P} / \mathrm{H}=3,0 \pm 2^{3} \text { ) }\end{array}$} & \multicolumn{3}{|c|}{$\begin{array}{l}\text { Gemischte } \\
\text { Biozönose } \\
\mathrm{P} / \mathrm{H}=20 \pm 4\end{array}$} & \multicolumn{3}{|c|}{$\begin{array}{l}\text { Autotrophe } \\
\text { Biozönose } \\
\mathrm{P} / \mathrm{H}=55 \pm 7\end{array}$} \\
\hline & Datum & & \multicolumn{3}{|c|}{ 17./18. August 1966} & \multicolumn{3}{|c|}{ 22./23. Juni 1966} & \multicolumn{3}{|c|}{ 8. April 1966} \\
\hline & $Q$ & $1 \mathrm{~s}^{-1}$ & 8 & 4 & 1 & 8 & 4 & 1 & 8 & 4 & 1 \\
\hline & Pegelstand & $\mathrm{cm}$ & 15,8 & 16,5 & 15,7 & 14,8 & 15,7 & 15,3 & 17,0 & 16,4 & 17,3 \\
\hline & Fliessgeschwindigkeit & $\mathrm{cm} / \mathrm{s}$ & 24 & 13 & 4 & 28 & 14 & 4 & 19 & 8 & 3 \\
\hline & Länge der Selbstreinigungsstrecke & $\mathrm{m}$ & 100 & 100 & 100 & 85 & 85 & 85 & 100 & 100 & 100 \\
\hline & Temperatur & ${ }^{\circ} \mathrm{C}$ & 12,7 & 12,7 & 13,5 & 10,3 & 10,7 & 12,5 & 7,4 & 7,9 & 8,2 \\
\hline & Sauerstoff $\mathrm{mg} O / 1$ & Anfang & 3,8 & 3,4 & 3,5 & 7,9 & 7,7 & 7,8 & 8,4 & 6,5 & 7,0 \\
\hline & & Ende & 4,9 & 4,8 & 5,4 & 8,4 & 8,5 & 9,8 & 9,8 & 9,8 & 18,7 \\
\hline & Bewuchsfläche & $\mathrm{m}^{2} / \mathrm{m}$ & & 0,51 & & & 0,55 & & & 0,52 & \\
\hline & Biomasse $^{1}$ ) & $\mathrm{g} \mathrm{TS} / \mathrm{m}^{2}$ & & $84,1 \pm$ & 43,3 & & $1,8 \pm$ & & & $1 \pm 15,5$ & \\
\hline & & $\mathrm{g} \mathrm{GV} / \mathrm{m}^{2}$ & & $76,5 \pm$ & 39,2 & & $2,2 \pm$ & & & $0 \pm 8,5$ & \\
\hline & & $\mathrm{g}$ org. $\mathrm{C} / \mathrm{m}^{2}$ & & 30,6 & & & $3,4 \pm$ & & & $9 \pm 4,0$ & \\
\hline \multirow[t]{5}{*}{ Glukose } & $s_{0}$ & $\mu M / 1$ & 1,13 & 1,17 & 1,24 & 2,67 & 1,81 & 0,66 & 1,36 & 1.67 & 1,34 \\
\hline & $s_{z}$ & $\mu M / 1$ & 0,29 & 0,46 & 0,76 & 1,68 & 1,30 & 0,45 & 1,33 & 1,64 & 1,21 \\
\hline & $\Delta s$ & $\mu M / 1$ & 0,84 & 0,71 & 0,48 & 0,99 & 0,51 & 0,21 & 0,03 & 0,03 & 0,13 \\
\hline & $Q \cdot \Delta s=S m$ & $\mu M / \mathrm{s}$ & 6,72 & 2,84 & 0,48 & 7,92 & 2,04 & 0,21 & 0,24 & 0,12 & 0,13 \\
\hline & $\left.S_{e}^{2}\right)$ & $10^{-9} \mathrm{M} / \mathrm{g} \mathrm{s}$ & 1,72 & 0,73 & 0,12 & 4,01 & 1,03 & 0,11 & 0,16 & 0,08 & 0,09 \\
\hline
\end{tabular}




\begin{tabular}{|c|c|c|c|c|c|c|c|c|c|c|}
\hline \multirow[t]{5}{*}{ Fructose } & so & 0,91 & 0,89 & 0,94 & 1,92 & 1,30 & 0,53 & 1,0 & 1,21 & 0,99 \\
\hline & $s_{z}$ & 0,27 & 0,37 & 0,60 & 1,17 & 0,89 & 0,32 & 0,98 & 1,18 & 0,89 \\
\hline & $\Delta s$ & 0,64 & 0,52 & 0,34 & 0,75 & 0,41 & 0,21 & 0,02 & 0,03 & 0,10 \\
\hline & $Q \cdot \Delta s$ & 5,12 & 2,08 & 0,34 & 6,0 & 1,64 & 0,21 & 0,16 & 0,12 & 0,10 \\
\hline & $\left.S_{e}^{2}\right)$ & 1,31 & 0,53 & 0,09 & 3,04 & 0,83 & 0,11 & 0,10 & 0,08 & 0,06 \\
\hline \multirow[t]{5}{*}{ Saccharose } & $s_{0}$ & 4,54 & 4,60 & 4,97 & 11,13 & 7,43 & 2,65 & 5,50 & 6,68 & 5,44 \\
\hline & $s_{z}$ & 1,32 & 1,96 & 3,10 & 7,0 & 5,38 & 1,81 & 5,38 & 6,48 & 4,91 \\
\hline & $\Delta s$ & 3,22 & 2,64 & 1,87 & 4,13 & 2,05 & 0,84 & 0,12 & 0,20 & 0,53 \\
\hline & $Q \cdot \Delta s$ & 25,76 & 10,56 & 1,87 & 33,04 & 8,20 & 0,84 & 0,96 & 0,80 & 0,53 \\
\hline & $\left.S_{e}^{2}\right)$ & 6,60 & 2,71 & 0,48 & 16,74 & 4,15 & 0,42 & 0,64 & 0,53 & 0,35 \\
\hline \multirow[t]{4}{*}{ Glutaminsäure } & $s_{0}$ & 4,54 & 4,74 & 4,98 & 9,20 & 9,37 & 3,05 & 9,78 & 7,04 & 10,66 \\
\hline & $s_{z}$ & 2,75 & 2,94 & 3,64 & 8,14 & 8,29 & 2,49 & 9,65 & 6,85 & 9,26 \\
\hline & $\Delta s$ & 1,79 & 1,80 & 1,34 & 1,06 & 1,08 & 0,56 & 0,13 & 0,19 & 1,40 \\
\hline & $\left.S_{e}^{2}\right)$ & 3,67 & 1,84 & 0,34 & 4,30 & 2,19 & 0,28 & 0,69 & 0,50 & 0,92 \\
\hline
\end{tabular}

$s_{0}=$ Konzentration am Anfang der Selbstreinigungsstrecke; $s_{z}=$ Konzentration am Ende der Selbstreinigungsstrecke; ${ }^{1}$ ) Artenzusammensetzung vgl. Tabelle 3. 2) Berechnet mit organ. Anteil (GV) der Biomasse. ${ }^{3}$ ) $\mathrm{P} / \mathbf{H}=$ Verhältnis von phototropher zu heterotropher Biomasse, berechnet als relativer Chlorophyllgehalt.

$s_{0}=$ Concentration at the beginning of the selfpurification stretch; $s_{z}=$ concentration at the end of the selfpurification stretch. 1 ) For a list of species see Table 3. ${ }^{2}$ ) Calculated on the basis of the organic matter in the biomass (loss on ignition). ${ }^{3}$ ) $\mathrm{P} / \mathrm{H}=\mathrm{Ratio}$ of phototrophic and heterotrophic biomass on the basis of relative chlorophyll-content. 
den Verunreinigungskomponenten wurde an den Versuchstagen kontinuierlich zugesetzt, dann stellte man die 3 Fliessgeschwindigkeiten nacheinander während je einer Stunde ein. In dieser Zeit wurden am Anfang und Ende der Teststrecke (Länge $=100 \mathrm{~m}$, im Versuch mit Mischbiozönose nur $85 \mathrm{~m}$ ) in Abständen von 15 Minuten Proben (total 4 Proben/Geschwindigkeit) erhoben. Da alle drei Fliessgeschwindigkeiten in derselben Teilstrecke am gleichen Tag geprüft wurden, waren die ökologischen Bedingungen für alle Proben gleichartig, so dass für jede Biozönose $3 \times 4 \times 2=$ 24 Einzelproben für den Vergleich der Strömungsgeschwindigkeiten zur Verfügung standen. Da alle Analysen mit 4 Parallelen vorgenommen wurden, besitzt die Gesamtheit der Werte 95 Freiheitsgrade. Die Bestimmung der Biomasse in der Prüfstrecke erfolgte nach Versuchsabschluss. In Abständen von etwa $10 \mathrm{~m}$ wurden 8 Teilstrecken von $1 \mathrm{~m}$ Länge völlig ausgeräumt und die abgeerntete Biomasse mit den auch früher verwendeten Parametern charakterisiert.

\subsection{Versuchsergebnisse}

Die analytischen Daten (Mittelwerte der 4 Parallelen pro Probe) sowie die spezifischen ökologischen Bedingungen an den Versuchstagen sind in Tabelle 4 zusammengefasst. Abb. 3 stellt die Werte für die spezifische Selbstreinigungsleistung der ge-

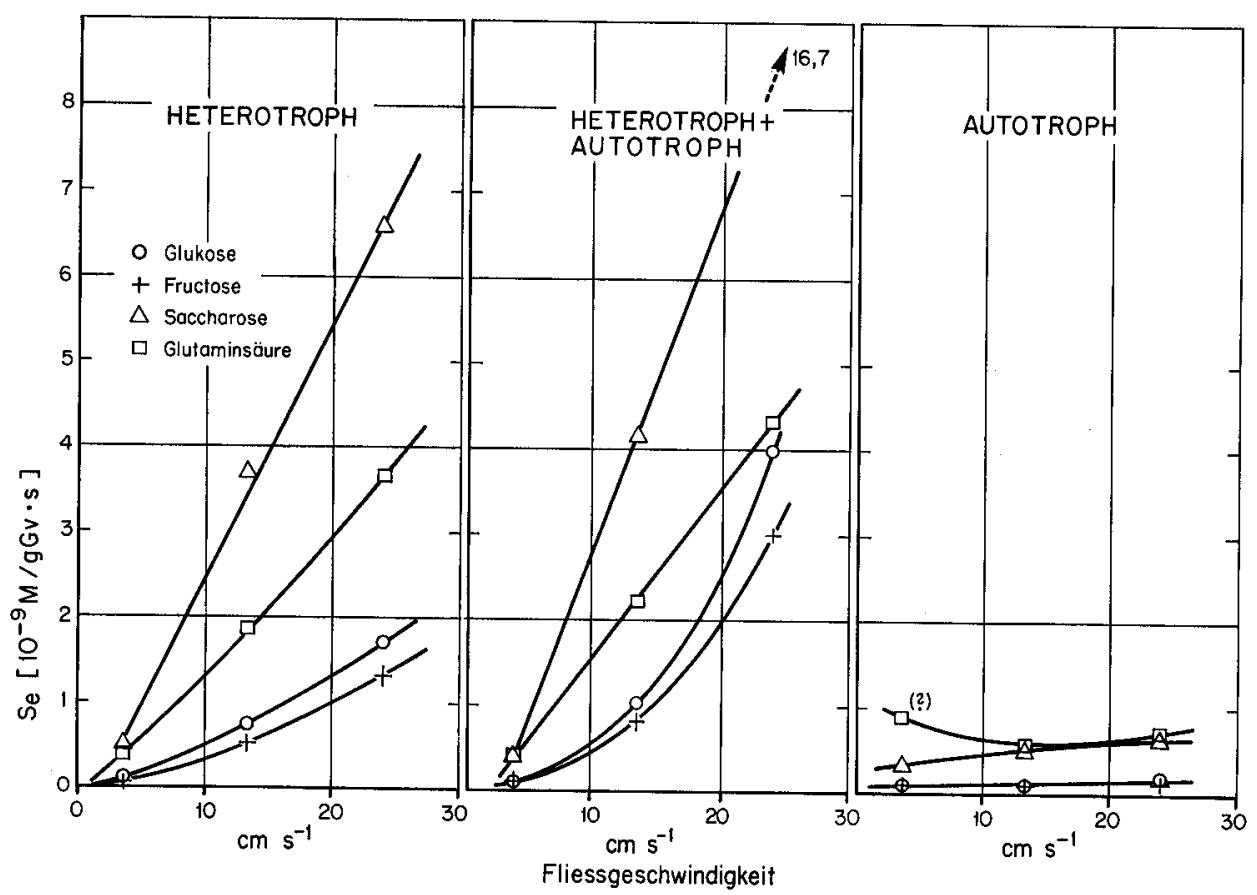

Abb. 3. Spezifische Selbstreinigungsleistungen von drei Biozönosetypen für verschiedene Einzelsubstrate in Funktion der Fliessgeschwindigkeit. Experimentelle Einzelheiten vgl. Tcxt und Tabelle 4.

Fig. 3. Specific elimination rate by the biomass for three types of biocenoses in function of the flow velocity. For experimental details see text and Table 4. 
prüften Biozönosen in Funktion der Fliessgeschwindigkeit dar $\left(S_{\boldsymbol{e}}\right.$ als $\mu M$ Substrat/g $G V \cdot s)$.

Die Zusammenfassung in Abb. 3 bedarf keines Kommentars. Es ist offensichtlich, dass in Bestätigung früherer Befunde die rein autotrophe Phytozönose keine ins Gewicht fallende Selbstreinigungsleistung erbringt. Dementsprechend ist auch kein Einfluss der Strömungsgeschwindigkeit auf das Konzentrationsverhalten der Verunreinigungssubstanzen zu bemerken. Wir werden auf dieses Experiment nicht mehr zurückkommen, da es im gegenwärtigen Kontext ohne Interesse ist. Wesentlich aufschlussreicher sind die Versuche mit der heterotrophen bzw. der gemischten heterotrophen-phototrophen Phytozönose: Entgegen der Erwartung steigt ihre Selbstreinigungsleistung mit zunehmender Fliessgeschwindigkeit, d.h. mit abnehmender absoluter Kontaktzeit des Wassers mit der Biomasse, gewaltig an. Ferner erreicht die gemischte Phytozönose bei $13 \mathrm{~cm} \mathrm{~s}^{-1}$ und $24 \mathrm{~cm} \mathrm{~s}^{-1}$ sogar höhere $S_{e}$-Werte als die rein heterotrophe Biomasse. Da die absolute Menge heterotropher Mikrophyten pro Rinnenstrecke in der gemischten Biozönose erheblich geringer war als in der rein heterotrophen Rinne, ist auch dieses Ergebnis keineswegs selbstverständlich.

In einem kontinuierlichen Fermentationssystem mit vorgegebenen äusseren Ökofaktoren sind die von der Biozönose resorbierten Substratmengen proportional zur Zeit und zur Menge der Biomasse und unter Umständen auch von der Substratkonzentration abhängig. Die unerwarteten experimentellen Ergebnisse sind daher nur kinetisch zu verstehen.

\subsection{Interpretation der experimentellen Beobachtungen}

Um den Einfluss der Fliessgeschwindigkeit auf die Selbstreinigungsleistung in der Teststrecke zu beurteilen, müsste man auf Grund der Resorptionseigenschaften der beteiligten Organismen, der vorhandenen Biomassemenge und des im Längsprofil aufgetretenen Substratgradienten eine ideale maximale Selbstreinigungsgeschwindigkeit berechnen und sie dann mit der im Experiment beobachteten Geschwindigkeit vergleichen.

\subsection{Grundlagen}

Der logische Ansatz für diese Aufgabe ergibt sich aus dem Verständnis einer Selbstreinigungsstrecke als eines kontinuierlichen Röhrenfermenters mit festsitzender Biomasse. Dabei ist daran zu denken, dass das natürliche System eines Flusses in mancher Hinsicht von den Bedingungen abweicht, welche für die Gültigkeit der mathematischen Theorie eines idealen Fermentationssystems Voraussetzung sind. Solche nichtidealen Bedingungen sind beispielsweise: heterogene räumliche Verteilung der Biomasse im System, nicht genau definierbare Strömungsbedingungen, Artenschichtung der Biomasse in Quer- und Längsprofil usw. Der prinzipielle Gedankengang der Theorie ist aber trotzdem anwendbar, so dass man versuchen kann, wenigstens in qualitativer Hinsicht die beobachteten Vorgänge richtig zu interpretieren.

Eine allgemeine Theorie des Röhrenfermenters ist bis jetzt nicht veröffentlicht worden. Wir haben uns in anderem Zusammenhang jedoch mit der algebraischen Lösung der an sich trivialen Differentialgleichung für dieses System beschäftigt (Imboden und Wuhrmann 1972, unveröffentlicht), und wir können auf diese Lösung 
zurückgreifen (vgl. Anhang). Die Konzentrationsabnahme eines Substrates dank des mikrobiellen Verbrauchs in Funktion der Fliessdistanz in einem idealen Röhrenfermenter ist gegeben durch die spezifische Resorptionsgeschwindigkeit der Biomasse, die Biomassemenge und die Fliessgeschwindigkeit (d.h. die Kontaktzeit zwischen Substratlösung und Biomasse). Die allgemeine Gleichung für den Substratgradienten ergibt sich aus

$$
\frac{d s}{d t}=-f(s)\left[x_{0}+Y\left(s_{0}-s_{z}\right)\right]
$$

worin der Faktor $x_{0}+Y\left(s_{0}-s_{z}\right)$ den Gradienten der Biomassekonzentration im Längsprofil auf der Fliessdistanz $z$ wiedergibt und $f(s)$ die Veränderung der Resorptionsgeschwindigkeit im Längsprofil infolge der abnehmenden Substratkonzentration beinhaltet.

Die Integration der Gleichung (2) ergibt:

worin

$$
V_{m} \frac{z}{p}=\ln \left[\left(\frac{x_{z}}{x_{0}}\right)^{\alpha+(1 / Y)} \cdot\left(\frac{s_{0}}{s_{z}}\right)^{\alpha}\right],
$$

$$
\alpha=-\frac{K_{m}}{x_{0}+Y s_{0}} .
$$

Betrachtet man eine Fließstrecke beschränkter Länge (z.B. 10 oder $20 \mathrm{~m}$ ) in einem Fluss, so wird in der Regel die Veränderung der stehenden Biomasse gering und schon aus Gründen der Ungleichmässigkeit der Sohlenbesiedlung kaum statistisch zu sichern sein. In erster Näherung darf man deshalb die Biomasseproportion $x_{z} / x_{0}$ als 1 annehmen, wodurch sich Gleichung (3) erheblich vereinfacht. Die uns interessierende Grösse der Veränderung der Substratkonzentration im Längsprofil wird dann

$$
\ln \frac{s_{z}}{s_{0}}=-\frac{V_{m}}{\alpha} \frac{z}{v} .
$$

Bei Kenntnis der "wahren" Werte von $V_{m}$ und $K_{m}$ der heterotrophen Organismengesellschaften im betrachteten Flussabschnitt können wir mit Gleichung (4) also ein theoretisch zu erwartendes Verhältnis von $s_{z} / s_{0}$ für eine bestimmte Fliessdistanz (bzw. Fliesszeit) bei gegebener Fliessgeschwindigkeit berechnen. Dieser Berechnungswert lässt sich dem im Naturexperiment gefundenen Ergebnis gegenüberstellen, und allfällige Unterschiede müssten dann auf die Beeinflussung der $V_{m^{-}}$und $K_{m}$-Werte durch den Parameter Fliessgeschwindigkeit zurückzuführen sein.

\subsection{Quantitative Ergebnisse über die Wechselwirkung von Strömung und Selbstreinigung}

Wir können nun die Ergebnisse der in Abschnitt 4 beschriebenen Konzentrationsversuche mit jenen der vorstehenden Experimente mit verschiedenen Fliessgeschwindigkeiten kombinieren. Dies ist zulässig, weil die externen ökologischen Faktoren praktisch identisch waren (Temperatur, Grundwasserchemismus, Art der dominanten Heterotrophen). Aus dem Konzentrationsexperiment (Tab. 2) übernehmen wir als Globalwerte von $V_{m}$ und $K_{m}$ die physiologisch günstigsten Werte $V_{m}^{*}$ und $K_{m}^{*}$ an Ende der Selbstreinigungsstrecke. Führen wir sie in Gleichung (4) ein, so 
können wir für die verschiedenen untersuchten Fliessgeschwindigkeiten $\boldsymbol{v}$ die Sollkonzentrationen der Substrate $s_{z}$-Soll berechnen, welche bei idealen Kontaktverhältnissen zwischen Biomasse und Flusswasser nach der Fliessdistanz $z=100 \mathrm{~m}$ hätten gefunden werden müssen. In Tabelle 5 sind diese theoretisch zu erwartenden Endkonzentrationen in der fliessenden Welle den effektiven Beobachtungen gegenübergestellt. Die logische Sequenz der Sollwerte ist unverkennbar: Wie es die Theorie

Tabelle 5. Erwartungswerte und beobachtete Werte für die Substratkonzentrationen nach $100 \mathrm{~m}$ Fließstrecke.

Table 5. Anticipated values and values observed for substrate concentrations after $100 \mathrm{~m}$ of flow distance.

\begin{tabular}{|c|c|c|c|c|c|c|c|c|c|}
\hline \multirow{2}{*}{$\begin{array}{l}\text { Substrat } \\
\nu\left[\mathrm{cm} \mathrm{s}^{-1}\right]\end{array}$} & \multicolumn{2}{|c|}{ Glukose } & \multicolumn{3}{|c|}{ Fructose } & \multicolumn{4}{|c|}{ Saccharose } \\
\hline & 24 & 13,3 & 3,7 & 24 & 13,3 & 24 & 24 & 13,3 & 3,7 \\
\hline $\ln \frac{s_{z}}{s_{0}}$ & $-3,16$ & $-5,71$ & $>-20$ & $-2,99$ & $-5,41$ & $>-20$ & $-2,92$ & $-5,26$ & $>-20$ \\
\hline so beob. $\mu \mathrm{g} \mathrm{C} / 1$ & 81,4 & 84,2 & 89,3 & 65,5 & 64,1 & 67,7 & 653,8 & 662,4 & 715,7 \\
\hline$s_{z}$ Soll & 3,4 & 0,3 & $\sim 0$ & 3,3 & 0,3 & $\sim 0$ & 35,4 & 3,4 & $\sim 0$ \\
\hline$\left.s_{z \text { beob. }}{ }^{1}\right)$ & 20,9 & 33,1 & 54,7 & 19,4 & 26,6 & 43,2 & 190,1 & 282,2 & 446,4 \\
\hline
\end{tabular}

1) Aus Tabelle 4.

fordert, nehmen sie mit zunehmender Fliessgeschwindigkeit (d.h. abnehmender Kontaktzeit zwischen Substraten und Biomasse) ab. Bei der kleinsten Fliessgeschwindigkeit hätten wir in unseren Experimenten nach $100 \mathrm{~m}$ Fließstrecke eine 100prozentige Selbstreinigung bezüglich der geprüften Substanzen feststellen müssen.

Auf Grund der zu erwartenden und der effektiv gemessenen Konzentrationsveränderungen kann man entsprechende Grössen der Selbstreinigung $S_{m}$-effektiv und $S_{m}$-Soll ermitteln und die prozentuale, auf die Fliessgeschwindigkeit zurückzuführende Veränderung der Selbstreinigung abschätzen (Tab. 6). Dieselbe Gegenüberstellung lässt sich auch bezüglich der Werte für die spezifische Selbstreinigungsleistung der Biomasse $S_{e}$ vornehmen, wobei man Tabelle 7 erhält.

Tabellen 6 und 7 zeigen, dass der Stoffumsatz einer vorgegebenen Biomasse mit abnehmender Fliessgeschwindigkeit stark vermindert wird (etwa auf die Hälfte, wenn

Tabelle 6. Relative Verminderung der Grösse der Selbstreinigung $S_{m}$ bei abnehmender Fliessgeschwindigkeit $(\mathrm{L}=100 \mathrm{~m})$.

Table 6. Relative reduction in the amount of selfpurification $S_{m}$ in relation to decreasing flow velocity $(\mathrm{L}=100 \mathrm{~m})$.

\begin{tabular}{|c|c|c|c|c|c|c|c|c|c|}
\hline \multirow[b]{2}{*}{$v\left[\operatorname{cm~s}^{-1}\right]$} & \multicolumn{3}{|c|}{$\begin{array}{l}{\left[S_{m} \text { effektiv }\right]} \\
\text { Rinnen-Exp. }\end{array}$} & \multicolumn{3}{|c|}{$\left[S_{m \text { Soll }}\right]$} & \multicolumn{3}{|c|}{$\begin{array}{c}\%\left[S_{m} \text { efpektiv }\right] \text { von } \\
{\left[S_{m} \text { Soll }\right]}\end{array}$} \\
\hline & 24 & 13,3 & 3,7 & 24 & 13,3 & 3,7 & 24 & 13,3 & 3,7 \\
\hline$\left[\mu M \mathrm{~s}^{-1}\right]$ & 6,72 & 2,84 & 0,48 & 8,64 & 4,66 & 1,24 & 78 & 61 & 39 \\
\hline$\left[\mu M \mathrm{~s}^{-1}\right]$ & 5,12 & 2,08 & 0,34 & 6,92 & 3,54 & 0,94 & 74 & 59 & 36 \\
\hline Saccharose $\left[\mu M \mathrm{~s}^{-1}\right]$ & 25,76 & 10,56 & 1,87 & 38,28 & 18,31 & 4,97 & 67 & 13,3 & 3,7 \\
\hline
\end{tabular}


Tabelle 7. Relative Verminderung der spezifischen Selbstreinigungsgeschwindigkeit der Biomasse durch Abnahme der Strömungsgeschwindigkeit. Mittelwert für $100 \mathrm{~m}$ Fliessdistanz.

$\mathrm{T}=12-13^{\circ} \mathrm{C}$.

Table 7. Relative reduction in the biomass' specific selfpurification rate as a result of reduced flow velocity. Mean value for $100 \mathrm{~m}$ flow distance. Temperature $=12-13^{\circ} \mathrm{C}$.

\begin{tabular}{lccc}
\hline$v\left[\mathrm{~cm} \mathrm{~s}^{-1}\right]$ & $100 \frac{V}{V_{m} *}=100 \frac{S_{e \text { effektiv }}}{S_{e \text { Soll }}}$ & & \\
& Glukose & Fructose & Saccharose \\
\hline 24 & 16 & 11 & 32 \\
13,3 & 6,9 & 4,3 & 13 \\
3,7 & 1,1 & 0,7 & 2,3 \\
\hline
\end{tabular}

$\nu$ von $24 \mathrm{~cm} \mathrm{~s}^{-1}$ auf $4 \mathrm{~cm} \mathrm{~s}^{-1}$ abnimmt). Die Verminderung kann nur durch eine erhebliche Herabsetzung der Substratkonzentration an den Rezeptorstellen der einzelnen Zellen zustande kommen. Eine solche kann aber nur aus der Geschwindigkeitsdifferenz der Substratkonsumation innerhalb von Organismenverbänden und der Transportgeschwinaigkeit der Substrate aus der fliessenden Welle an die Rezeptorstellen entstehen, d.h. sie ist eine Folge extrazellulärer Faktoren. Man kann diese Argumentation rechnerisch überprüfen, da wir ja in der Beziehung (2) für $V, V_{m}^{*}$ und $K_{m}^{*}$ experimentelle Beobachtungen besitzen. Wir können also die effektiv wirksame Substratkonzentration $s$ an den Rezeptoren der Einzelzellen im Sinne eines globalen Mittelwertes für die gesamte heterotrophe Biomasse schätzen, welche für das Zustandekommen der gemessenen spezifischen Selbstreinigungsleistung $V$ notwendigerweise vorhanden sein müsste. Aus (2) ergibt sich

$$
s_{\text {Rezeptoren }}=\frac{V / V_{m}}{1+V / V_{m}} K_{m} .
$$

Wenn wir die berechnete Konzentration $s_{\text {Rezeptoren }}$ den gemessenen Konzentrationen $s_{z}$ der Substrate in der fliessenden Welle gegenüberstellen, muss sich der mittlere Substratgradient zwischen freiem Wasser und der Gesamtheit der Zelloberflächen zeigen. Das Verhältnis zwischen den beiden Konzentrationen sollte bei gleichen Fliessgeschwindigkeiten für alle Substrate annähernd dasselbe sein. Tabelle 8 enthält für zwei Teilstrecken $(0-10 \mathrm{~m}$ und $90-100 \mathrm{~m})$ die Berechnungsresultate nach Beziehung (5). Mit Ausnahme eines einzigen Wertes ist das Ergebnis überraschend homogen, denn tatsächlich zeigen sich für gleiche ökologische Bedingungen annähernd gleiche Proportionen $s_{\text {Rezeptoren }} / s_{\text {wasser. }}$ Man findet gemäss dieser Abschätzung, dass in den beiden Teilstrecken für alle Substrate bei der Fliessgeschwindigkeit von $24 \mathrm{~cm}$ $\mathrm{s}^{-1}$ an den Zelloberflächen im Mittel etwa die Hälfte der Substratkonzentration herrscht, welche in der fliessenden Welle vorhanden ist; bei $13 \mathrm{~cm} \mathrm{~s}^{-1}$ sind es noch etwa $12-14 \%$ und bei $3,7 \mathrm{~cm} \mathrm{~s}^{-1}$ noch rund $1 \%$. Damit ist die vorherige Frage nach der Ursache der raschen Abnahme des Stoffumsatzes der Biozönosen mit abnehmender Strömungsgeschwindigkeit zumindest in qualitativer Hinsicht ausreichend beantwortet. Die vorstehenden Konzentrationsangaben sind Rechnungsergebnisse und gelten als statistische Grösse für die Oberfläche der Gesamtheit der heterotrophen Biomasse im Gerinne. Wie es sich mit den effektiven Oberflächenkonzentrationen 
bei einzelnen Zellen in verschiedenen Schichten der Biozönose verhält, bleibt selbstverständlich eine offene Frage.

Tabelle 8. Abschätzung der effektiven mittleren Substratkonzentration an den Rezeptoren der heterotrophen Komponenten der Rinnenphytozönosen.

Table 8. Estimate of the actual mean substrate concentration at the receptor sites of the heterotrophic components of the phytocoenoses in the river model.

\begin{tabular}{|c|c|c|c|c|c|c|c|c|c|}
\hline \multirow[b]{3}{*}{$\nu\left[\mathrm{cm} \mathrm{s}^{-1}\right]$} & \multicolumn{9}{|c|}{ Substrat } \\
\hline & \multicolumn{3}{|c|}{ Glukose } & \multicolumn{3}{|c|}{ Fructose } & \multicolumn{3}{|c|}{ Saccharose } \\
\hline & 24 & 13 & 3,7 & 24 & 13 & 3,7 & 24 & 13 & 3,7 \\
\hline \multicolumn{10}{|l|}{ Teilstrecke $0-10 \mathrm{~m}$} \\
\hline $\bar{s}$ berechnet $\mu \mathrm{g} \mathrm{C} / \mathrm{l}$ & 40,8 & 11,6 & 1,4 & 26,2 & 8,6 & 1,2 & 481 & 104 & 12,8 \\
\hline$\mu g \mathrm{C} / 1$ & 76,1 & 80,4 & 87,3 & 61,8 & 61,4 & 68,1 & 615,7 & 635 & 699 \\
\hline $\bar{s}_{\text {ber }} / \bar{s}_{\text {exp }}$ & 53 & 14 & 1,6 & 42 & 14 & 1,7 & $(78) ?$ & 16 & 1,8 \\
\hline \multicolumn{10}{|l|}{ Teilstrecke 90-100 m } \\
\hline $\bar{s}$ berechnet $\mu \mathrm{g} \mathrm{C} / 1$ & 8,9 & 4,4 & 0,9 & 7,9 & 3,4 & 0,8 & 90,9 & 42,3 & 8,1 \\
\hline$\mu \mathrm{g} C / 1$ & 22,3 & 34,7 & 56,0 & 20,6 & 27,8 & 44,2 & 202,5 & 294,8 & 457,1 \\
\hline $\bar{s}_{\text {ber. }} / \bar{s}_{\exp }$. & 40 & 73 & 7,6 & 38 & 12 & 1,7 & 45 & 14 & 1,8 \\
\hline
\end{tabular}

Grundlage: $x=156 \mathrm{~g} \mathrm{C} /$ Teilstrecke; $V_{m}^{*}$ und $K_{m}^{*}$ gemäss Tabelle $2 ; \bar{s}_{\text {ber. }}=\frac{V / V_{m}^{*}}{1+V / V_{m}^{*}} K_{m}^{*}$;

$\bar{s}_{\text {exp. }}=$ aus Messwerten in der fliessenden Welle mittels $e$-Funktion errechnet.

Basis: $x=156 \mathrm{~g} \mathrm{C} / \mathrm{stretch} ; V_{m}^{*}$ and $K_{m}^{*}$ according to Table $2 ; \bar{s}_{\text {ber. }}=\frac{V / V_{m}^{*}}{1+V / V_{m}^{*}} K_{m}^{*}$;

$\bar{s}_{\text {exp. }}=$ values measured in the flowing wave calculated on the basis of the $e$ function.

Das zunächst qualitativ zu beurteilende Ergebnis der Konzentrationsverhältnisse der Substrate verleitet zu einer weiteren Interpretation der Selbstreinigungsbeobachtungen hinsichtlich der Wachstumgsgeschwindigkeit und Biomasseproduktion der heterotrophen Biozönosekomponenten, insbesondere auch im Falle gemischter heterotropher-phototropher Gemeinschaften, bei welchen die Dominanzverhältnisse in einer Selbstreinigungsstrecke im wesentlichen von den Proportionen der Wachstumsgeschwindigkeiten der beiden Mikrophytengruppen bestimmt werden. Wir möchten uns in einer weiteren Arbeit mit diesen zusätzlichen Fragen befassen.

\section{Zusammenfassung}

Die Selbstreinigungsleistung verschiedener Biozönosetypen wurde bei Fliessgeschwindigkeiten von 24, 13 und 3,7 $\mathrm{cm} \mathrm{s}^{-1}$ unter sonst gleichartigen Bedingungen in Modellflüssen gemessen. Die spezifische Selbstreinigungsleistung der beobachteten heterotrophen Biozönosen vermindert sich mit abnehmender Fliessgeschwindigkeit des Wassers entgegen den Erwartungen ganz erheblich, unabhängig von der Art der zu eliminierenden Substrate. Messungen der Selbstreinigung bei verschiedenen Konzentrationen der gleichen Verunreinigungssubstanzen gestatteten die Er- 
mittlung globaler, kinetischer Parameter für die Substratresorption der gesamten Biomasse. Mit ihrer Hilfe konnte rechnerisch gezeigt werden, dass die Abnahme der Selbstreinigungsleistung und -geschwindigkeit bei Verminderung der Fliessgeschwindigkeit von der Erschwerung der Substrattransporte aus der fliessenden Welle an die Rezeptoren für aktive Transporte an den Organismenoberflächen verursacht wird.

\section{RÉSUMÉ}

L'influence de la vitesse du courant sur l'autoépuration dans les eaux courantes

L'activité autoépuratrice de đivers types de biocénoses a été mesurée dans des modèles de rivières aux vitesses du courant de 24,13 et $3,7 \mathrm{~cm} \mathrm{~s}^{-1}$ sous des conditions autrement identiques. L'activité spécifique d'élimination de la biomasse hétérotrophe diminue considérablement, contre toute attente, au fur et à mesure que la vitesse du courant est réduite, indépendamment du type de substrat à éliminer. Les mesures de l'autoépuration à diverses concentrations de mêmes substances contaminantes, ont permis de déterminer des paramètres cinétiques globaux pour la résorption de substrats par la biomasse totale. A l'aide de ces paramètres, il a été possible de démontrer que la diminution de la vitesse d'autoépuration et de l'activité d'élimination spécifique de la biomasse, à des petites vitesses de courant, était dûe à une limitation progressive du transfert des substrats de l'onde courantc aux récepteurs responsables du transport active à la surface des organismes.

\section{SUMMARY}

\section{Flow Velocity as a Rate Determining Factor for Selfpurification in Rivers}

The selfpurification rate of various types of biocoenoses was measured in model rivers at flow velocities of 24,13 and $3.7 \mathrm{~cm} \mathrm{~s}^{-1}$ with all other parameters remaining constant. Contrary to expectation, the specific selfpurification activity of the heterotrophic biocoenoses declines considerably as water flow velocity decreases, regardless of the type of substrate to be eliminated. Measurements of selfpurification activities at various concentrations of the same pollutant enable the determination of overall kinetic parameters for substrate resorption by the total biomass. These parameters could be used to show by calculation that the decrease of the rate of selfpurification and of the specific elimination activity of the biomass at lower water flow rates were due to the fact that the substrate transport from the flowing wave to the receptors on organism surfaces responsible for active uptake was considerably inhibited as the flow velocities were reduced.

\section{LITERATURVERZEICHNIS}

[1] Wuhrmann, K., Eichenberger, E., Krähenbühl, H. R., und Ruchti, J., Modelluntersuchungen über die Selbstreinigung in Fliessgewässern, Verh. int. Ver. Limnol. 16, 897-905 (1966).

[2] Wuhrmann, K., Ruchti, J., und EIchenberger, E., Quantitative Experiments on SelfPurification with Pure Organic Compounds, Third Int. Conf. Wat. Poll. Res., Adv, Water Poll. Res. 7, 229-251, WPCF Washington (1967).

[3] Wuhrmann, K., Effect of Oxigen Tension on Biochemical Reactions in Sewage Purification Plants, in: Adv. in Biological Waste Treatment (Hrsg. Eckenfelder und McCabe; Pergamon Press, Oxford 1963), S.27-40. 
[4] Wuhrmann, K., River Bacteriology and the Role of Bacteria in Selfpurification of Rivers, in: Principles and Applications in Aquatic Microbiology (Hrsg. Heukelekian und Dondero; Wiley, New York 1964).

[5] Ruchtr, J., und Kunkler, D., Enzymatische Bestimmung von Glucose, Fructose und Saccharose in Gewässern, Schweiz. Z. Hydrol. 28, 62-68 (1966).

Anschrift der Autoren:

Prof. Dr. K. Wuhrmann, Dr. E. Eichenberger, Dr. H. Leidner, Doris Wüest-Kunkler, EAWAG, $\mathrm{CH}-8600$ Dübendorf.

\section{Anhang}

Die Gleichungen (2) und (3) auf Seite 266, welche den Gradienten der Biomasse bzw. des zuwachsbegrenzenden Substrates in einem Röhrenfermenter beschreiben, leiten sich aus den herkömmlichen Grundannahmen der Theorie der kontinuierlichen Fermentationen wie folgt ab:

Wir betrachten zwei Querschnitte im Abstand $d z$ in unserem (laminar) durchflossenen Röhrenfermenter, wobei zuflußseitig die Substratkonzentration $s_{\mathbf{0}}$ bzw. die Biomassekonzentration $x_{0}$ vorhanden seien, die sich auf der Fließstrecke $d z z u s_{z}$ $\left(s_{z}<s_{0}\right)$ bzw. $x_{z}\left(x_{z}>x_{0}\right)$ verändern. Die Fliessgeschwindigkeit des Wassers sei $\nu$. Die Geschwindigkeit der Substrataufnahme $V$ durch die Biomasse (messbar als Geschwindigkeit der Abnahme des Massentransportes von $s$ ) sei enzymatisch reguliert nach dem Ansatz von Michaelis und Menten

$$
V=V_{m} \frac{s}{K_{m}+s}
$$

( $K_{m}=$ Halbwertkonzentration des Substrates für die Resorptionsgeschwindigkeit), und die Biomassebildung sei eine konstante Fraktion $Y$ (yield coefficient) des verbrauchten begrenzenden Substrates

$$
d x=-Y d s
$$

In einer Fließstrecke $d z$ mit der Fliesszeit $d t=d z / v$ beträgt dann die Substratzehrung $d s / d z$ der vorhandenen Biomasse $x$

$$
\frac{d s}{d z}=-\frac{V}{\nu} x=-f(s) x
$$

wobei

$$
f(s)=\frac{V_{m}}{v} \cdot \frac{s}{K_{m}+s} .
$$

Der Biomassezwwachs ist entsprechend Gleichung (2)

oder auch

$$
\frac{d x}{d z}=-Y \frac{d s}{d z}
$$

$$
x=x_{0}+Y\left(s_{0}-s\right)
$$


Dies, in Gleichung (3) eingesetzt, führt zu

$$
\frac{d s}{d z}=-f(s)\left[x_{0}+Y\left(s_{0}-s\right)\right]
$$

als allgemeine Differentialgleichung für den Röhrenfermenter.

Schreibt man Gleichung (5) als Integral, so erhält man

$$
-z=\frac{\nu}{V_{m}} \int_{s_{0}}^{s z} \frac{K_{m}+s}{s} \cdot \frac{1}{x_{0}+Y\left(s_{0}-s\right)} d s .
$$

Seine Lösung (mit Hilfe einer Substitution für $x_{0}+Y s_{0}$ ) ergibt

$$
-\frac{z}{\nu} V_{m}=\ln \left\{\frac{s_{z} x_{0}}{s_{0}\left[x_{0}+Y\left(s_{0}-s_{z}\right)\right]}\right\}^{K_{m} /\left(z_{0}+Y_{0}\right)}-\ln \left[\frac{x_{0}+Y\left(s_{O}-s_{z}\right)}{x_{0}}\right]^{1 / Y} .
$$

Nach Gleichung (4a) ist $x_{0}+Y\left(s_{0}-s_{z}\right)=x_{z}$, und wir setzen ferner

Man kann dann auch schreiben

$$
\alpha=\frac{K_{m}}{x_{0}+Y s_{O}} .
$$

oder

$$
\frac{z}{\nu} V_{m}=\ln \left\{\left(\frac{x_{z}}{x_{0}}\right)^{\alpha+(1 / Y)} \cdot\left(\frac{s_{0}}{s_{z}}\right)^{\alpha}\right\}
$$

$$
e^{(z / v) V_{m}}=\frac{\left(\frac{x_{z}}{x_{0}}\right)^{\alpha+(1 / Y)}}{\left(\begin{array}{c}
s_{z} \\
s_{0}
\end{array}\right)^{\alpha}}
$$

D. Imboden, K. Wuhrmann 